\title{
Diagnóstico del pensamiento crítico de estudiantes de educación primaria de Chimbote, Perú
}

\author{
Diagnosis of Critical Thinking of Elementary School Students in Chimbote, Peru \\ Diagnóstico do pensamento crítico dos alunos do ensino fundamental de Chimbote, Peru
}

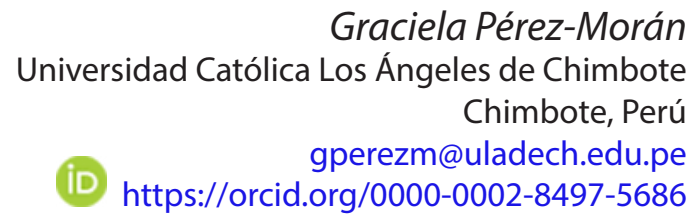

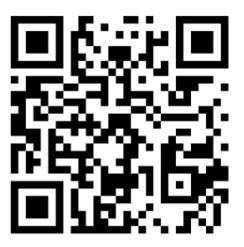

Janina Bazalar-Palacios

Universidad Católica Los Ángeles de Chimbote

Chimbote, Perú

jbazalarp@uladech.edu.pe

(D) http://orcid.org/0000-0001-8515-0742

Wendy Arhuis-Inca

Universidad Católica Los Ángeles de Chimbote

Chimbote, Perú

warhuisi@uladech.edu.pe

http://orcid.org/0000-0002-5317-5053

Recibido • Received • Recebido: 22 / 04 / 2019

Corregido • Revised • Revisado: 21 / 10 / 2020

Aceptado • Accepted • Aprovado: 04 / 12 / 2020

\begin{abstract}
Resumen:
Objetivo. Determinar el nivel de desarrollo del pensamiento crítico del estudiantado de las instituciones educativas de educación primaria de Chimbote. Materiales y métodos. Estudio descriptivo, se realizó en Chimbote, 2016. El pensamiento crítico fue evaluado en 250 niños y niñas que pertenecen al sexto grado de educación primaria en el año 2016. Se aplicó un cuestionario de 30 preguntas orientadas a abordar las dimensiones dialógica y sustantiva; cada pregunta incluía 5 opciones de respuesta en escala de Likert ( $1=$ total desacuerdo hasta $5=$ total acuerdo). Estas variables fueron recategorizadas mediante baremación por percentiles clasificados en tres categorías: (a) alto, entre 150-100 puntos; (b) medio, entre 99-50; y (c) bajo, entre 49-0 puntos. Se realizó un análisis descriptivo de la variable pensamiento crítico, según sus dimensiones sustantiva y dialógica, a través de tablas con sus frecuencias relativas y absolutas. Resultados. Existe una baja frecuencia en el desarrollo del pensamiento crítico, el cual está integrado por las dimensiones sustantiva y dialógica, donde el nivel bajo obtuvo más alto porcentaje con un $54 \%$ en comparación del nivel alto con $4,4 \%$. Conclusión. Existe un déficit de pensamiento crítico en el estudiantado y en sus dimensiones sustantiva y dialógica.
\end{abstract}

Palabras claves: Pensamiento crítico; niñez; dimensión dialógica; dimensión sustantiva; instituciones educativas. 
http://doi.org/10.15359/ree.25-1.15

http://www.una.ac.cr/educare

educare@una.ac.cr

\begin{abstract}
.
Objective: To determine the level of development of critical thinking of the students of the Educational Institutions of primary education in Chimbote, Peru. Materials and methods: A descriptive study was conducted in Chimbote, 2016. Critical thinking was evaluated in 250 boys and girls who belong to the sixth grade of primary education in 2016 . A questionnaire of 30 questions aimed at addressing the dialogic and substantive dimensions was applied; each question included five (5) response options on the Likert scale ( $1=$ total disagreement up to $5=$ total agreement). These variables were recategorized by scaling by percentiles classified into three categories: (a) high, between 150-100 points; (b) medium, between 99-50; and (c) low, between 49-0 points. A descriptive analysis of the critical thinking variable was carried out according to its substantive and dialogic dimensions through tables with their relative and absolute frequencies. Results: here is a negative tendency in developing critical thinking, which comprises the substantive and dialogical dimensions, being the lowest level the one that obtained the highest percentage of 54\%, compared to the high level of $4.4 \%$. Conclusion: There is a deficit of critical thinking in students and in their substantive and dialogical dimensions.
\end{abstract}

Keywords: Critical thinking; children; dialogical dimension; substantive dimension; educational institutions.

\title{
Resumo:
}

Objetivo. Determinar o nível de desenvolvimento do pensamento crítico de estudantes das Instituições Educacionais do Ensino Fundamental de Chimbote. Materiais e Métodos. A pesquisa foi um estudo descritivo, realizado em Chimbote, 2016. O pensamento crítico foi avaliado em 250 crianças matriculadas na $6^{a}$ série em 2016. Para isso, foi utilizado um questionario de 30 questões, distribuídas, englobando a dimensão dialógica e substantiva; cada questão incluia cinco opções de resposta escala de Likert ( 1 = discordo totalmente a $5=$ concordo), finalmente, re-categorizados por porcentuais em três categorias (alta, média e baixa). Foi feita uma análise descritiva da variável pensamento crítico, de acordo comsuas dimensões substantivas e dialógicas, através de tabelas com suas frequências relativas e absolutas. Resultados. Existe uma tendência negativa no desenvolvimento do pensamento crítico, que é integrado pelas dimensões substantiva e dialógica, onde o nível baixo teve a percentagem mais elevada, com 54\% em comparação com o nível alto de $4,4 \%$. Conclusão. Há um déficit de pensamento crítico nos estudantes e en suas dimensões substantivas e dialógicas.

Palavras chave: Pensamento crítico; infancia; dimensão dialógica; dimensão substantiva; instituições educativas.

\section{Introducción}

Desde los inicios de la nueva reforma educativa peruana, se ha incrementado el interés por promover un mayor desarrollo de las capacidades que permitan afrontar situaciones retadoras o problemáticas, a través de un "saber hacer"; y esto directamente conlleva al enfoque por competencias, que es la base actual de la educación a nivel mundial. Una serie de dominios que 
http://doi.org/10.15359/ree.25-1.15

dicho enfoque comprende, son: creatividad e innovación, pensamiento crítico, autoaprendizaje, comunicación, colaboración y trabajo en equipo, adaptabilidad, proactividad, orientación a resultados, liderazgo, responsabilidad y respeto (Bezanilla Albisua et al., 2018). Es decir, el pensamiento crítico es una macro habilidad compleja, pero necesarias en el estudiantado para un desarrollo de capacidades y dominios fundamentales en la educación (Díaz-Larenas et al., 2019).

Por otro lado, el Ministerio de Educación en Perú (2016a), basado en el enfoque por competencias, busca fomentar la comunicación, expresión, interacción, autonomía, valoración, desarrollo personal para convivir mejor y tener una buena calidad de vida. Ahora bien, el pensamiento crítico, como proceso de análisis, entendimiento y evaluación, es la fuente esencial para alcanzar dichos aprendizajes en cualquier tipo de área, ya sea curricular, e incluso extracurricular. Sin embargo, aunque las intenciones educativas del pensamiento crítico son englobar, de manera simple, sencilla, práctica e innovadora, la verdad es que los resultados demuestran lo contrario. Esto, porque uno de los indicadores que lo respaldan es aún prevalente en la práctica del modelo tradicional en muchos sectores de nuestra población.

La educación básica -base del crecimiento cognitivo- representa un serio problema en el Perú. El Programa Internacional para la Evaluación de Estudiantes (PISA) hace referencia a que Perú es el país que cuenta con un 54\% promedio bajo de desempeño, lo cual lo ubica en el antepenúltimo puesto en comprensión lectora a nivel mundial (Ministerio de Educación, 2015a).

Así mismo, es poco reconfortante que estudiantes de las instituciones educativas tan solo sepan leer, escribir y practicar la memorización frente a las nuevas informaciones. Esa situación conlleva al olvido de lo que en esencia se quiso aprender a aprender y quizás ni siquiera es procesada, analizada o evaluada, como bien podría hacerse con un pensamiento crítico. Paul y Elder (2003) afirman que el pensamiento de mala calidad es tan costoso, en un sentido económico, como el tipo de ritmo de vida que es llevada por las personas. Por tanto, la excelencia en el pensamiento depende de la puesta en ejercicio de este mismo, en forma sistemática.

Tamayo et al. (2015, p. 114) afirman que "[el proceso de] enseñanza-aprendizaje [debe conducir] a la constitución de pensamiento crítico [la cual exija] nuevas formas de entender las relaciones entre los estudiantes, los profesores y los saberes que circulan en las aulas de clase", así como también a desarrollar esa capacidad de inferir, argumentar, debatir, analizar, reflexionar en la búsqueda de solución de problemas.

En ese sentido, urge emprender políticas educativas de Estado mucho más efectivas para establecer reformas en el proceso de enseñanza-aprendizaje con calidad, en donde el estudiantado sea artífice de la construcción de sus aprendizajes y esté en constante búsqueda de nuevos conocimientos (López Aymes, 2012). Dicho esto, el pensamiento crítico, como proceso y como competencia, es esencial para encaminar a un individuo a ser competente dentro de la sociedad. 
Para ello, el Ministerio de Educación (MINEDU), en su afán de resolver el problema, ha creado las Rutas de aprendizaje (Ministerio de Educación, 2015b), donde precisa que el desarrollo del pensamiento crítico -capacidad de reflexionar, emitir juicios divergentes, extraer conclusiones, analizar críticamente y distinguir ideas de manera autónoma- (López Aymes, 2012) es fundamental para aminorar las cifras obtenidas por PISA.

Son múltiples las investigaciones que en la actualidad se han realizado en cuanto al pensamiento crítico en el Perú. En Lima, un hallazgo dirigido al nivel secundaria (Prado Coronado, 2019), mencionó que la mayoría de estudiantes alcanzaron puntajes en un nivel medio del pensamiento crítico, con puntajes demasiado cercanos al nivel bajo. Al igual que (Milla Virhuez, 2012), otro estudio -también en Lima- reveló que los grupos escolares de quinto año del nivel secundaria se ubicaron en un nivel promedio con $60,2 \%$ en tanto $28,3 \%$ se encuentra en un nivel bajo. En Ancash, el reporte del resultado (Ministerio de Educación, 2016b) de la evaluación censal de estudiantes (ECE), que mide la calidad de los aprendizajes, señala que en el indicador de lectura $28,3 \%$ de quienes cursan el cuarto grado de primaria se ubican en los niveles previo al inicio e inicio.

Es evidente que estas publicaciones han utilizado como unidad de análisis a estudiantes de educación secundaria y superior, ello denota la ausencia de estudios en escolares de educación inicial y primaria, etapas medulares donde se germina el ejercicio del razonamiento, análisis, reflexión y divergencias, para su continuo aprendizaje.

Esta problemática que afecta a la población peruana, en un contexto menor, también repercute en estudiantes que pertenecen a instituciones educativas de Chimbote, con presentan problemas para emitir juicios de valor, críticas constructivas o reflexivas, interpretación en los contenidos de información desde sus propios puntos de vista. En ese sentido, se determinó el nivel de desarrollo del pensamiento crítico del estudiantado en las instituciones educativas de educación primaria de Chimbote-Perú, 2017. Oportunidad sensata para obtener una visión real y objetiva del desarrollo de esta capacidad superior fundamental del pensamiento crítico (Villa y Poblete, 2007), y, a la vez, relevante porque contribuirá con un aporte científico donde el profesorado sea mejor preparado y capacitado en metodologías activas que redunden en elevar el pensamiento crítico y mejorar el desempeño en el aula (MINEDU, 2006).

\section{Método}

\section{Diseño y contexto del estudio}

Estudio descriptivo (Hernández Sampieri et al., 2014), se trata de un estudio primario con escolares de educación primaria de la ciudad de Chimbote, ubicada a orillas del océano Pacifico, ubicada a 373 kilómetros al norte de Lima, capital de Perú. Chimbote acoge un total de 112 colegios de EBR, de ellos 55 pertenecen a educación primaria y cuenta con 14236 estudiantes matriculados en primaria para el año 2017. 
http://doi.org/10.15359/ree.25-1.15

\section{Participantes}

El marco muestral estuvo constituido por la niñez matriculada en el $6^{\circ}$ grado de educación primaria -se eligió este grado debido a que están a puertas de ingresar a un nivel superior $y$, por ende, la dificultad en la comprensión es mayor- de las instituciones educativas públicas de la ciudad de Chimbote. Se incluyó a 250 estudiantes entre varones y mujeres con matrícula en el $6^{\circ}$ grado de educación primaria de 4 instituciones educativas públicas. La selección de las instituciones se realizó a partir de un muestreo por conveniencia, debido a la privación de los permisos para la ejecución del estudio, entre otros inconvenientes; y la de estudiantes se realizó mediante un muestreo aleatorio simple. Para llevar a cabo el estudio, primero se contó con el permiso de las instituciones educativas, luego la investigadora principal administró el instrumento a través de una prueba piloto a estudiantes fuera de la muestra de estudio $y$, posteriormente, se validó por personas expertas, en seguida, por medio de la prueba estadística, se mostró una alta confiabilidad reportando un indicador alpha de Cronbach de 0,89. Para el estudio en sí, se contó con el permiso de los padres y madres de familia del estudiantado, se obtuvo el consentimiento informado a fin de recibir autorización en el recojo de información. Luego se aplicó el cuestionario a estudiantes que asistieron el día de la aplicación.

\section{Instrumento}

El cuestionario que mide el nivel de desarrollo del pensamiento crítico se usó en el presente estudio, elaborado por Santiuste et al. (2001, citados por Marciales Vivas, 2003). El cuestionario consta de 30 preguntas orientadas a abordar las dimensiones dialógicas, análisis o integración de puntos de vista divergentes o en contraposición con el suyo propio (indicadores, lectura dialógica, escritura dialógica y escuchar-expresar oralmente dialógico) constituida por 22 preguntas; y sustantivas, se enfoca en el sustento del punto de vista del estudiantado (indicadores, lectura sustantiva, escritura sustantiva y escuchar-expresar oralmente sustantivo) por 8 preguntas.

\section{Variables}

Variable dependiente: Nivel de desarrollo de pensamiento crítico. “El pensamiento crítico [es] la complejidad y diversidad de los procesos cognitivos [(sustantivo y dialógica)] que lo integran, algunos más prototípicos de éste que otros, pero interactuando todos conjuntamente" (Marciales Vivas, 2003, p. 62).

El cuestionario de evaluación de pensamiento crítico contiene 30 preguntas, cada pregunta con 5 opciones de respuesta en escala de Likert (1=total desacuerdo hasta $5=$ total acuerdo). Estas variables fueron recategorizadas mediante baremación por percentiles clasificados en tres categorías: (a) alto, entre 150-100 puntos; (b) medio, entre 99-50; y (c) bajo, entre 49-0 puntos. 
http://doi.org/10.15359/ree.25-1.15

http://www.una.ac.cr/educare

educare@una.ac.cr

\section{Plan de análisis}

Para el análisis, los datos fueron ingresados a una base de datos de Microsoft Excel 2016, luego fueron exportados al software IMB SPSS Statistics v24.0 para su procesamiento. Se realizó un análisis descriptivo de la variable pensamiento crítico, según sus dimensiones sustantiva y dialógicas; se presentaron en tablas con sus frecuencias relativas y absolutas.

\section{Consideraciones éticas}

Este proyecto de investigación fue revisado y evaluado por el Comité de Ética de la Universidad Católica los Ángeles de Chimbote (CIE-ULADECH Católica) previamente a su ejecución y aprobado según informe de autorización: №004-2016-CEI-VI-ULADECH-Católica.

\section{Resultados}

\section{Nivel de desarrollo del pensamiento crítico del estudiantado}

Existe una tendencia negativa en el desarrollo del pensamiento crítico, el cual está integrado por las dimensiones sustantiva y dialógica, el nivel bajo obtuvo más alto porcentaje con un 54\% en comparación del nivel alto con 4,4\%. Ver Tabla 1.

Tabla 1: Nivel de desarrollo del pensamiento crítico del estudiantado

\begin{tabular}{ccc}
\hline Pensamiento crítico & $\mathrm{n}$ & $\%$ \\
\hline Alto & 11 & 4,4 \\
Medio & 103 & 41,2 \\
Bajo & 136 & 54,4 \\
\hline Total & 250 & $100 \%$ \\
\hline
\end{tabular}

Nota: Elaboración propia.

\section{Nivel de desarrollo del pensamiento crítico sustantivo del estudiantado}

En la Tabla 2 se muestra los resultados de acuerdo con la dimensión sustantiva del pensamiento crítico del estudiantado, de igual manera existe mayor proporción en el nivel bajo $65,6 \%$, respecto al nivel alto con $2,8 \%$. 
http://doi.org/10.15359/ree.25-1.15

Tabla 2: Nivel de pensamiento crítico sustantivo del estudiantado

\begin{tabular}{crc}
\hline Sustantivo & $\mathrm{n}$ & $\%$ \\
\hline Alto & 7 & 2,8 \\
Medio & 79 & 31,6 \\
Bajo & 164 & 65,6 \\
\hline Total & 250 & $100 \%$ \\
\hline
\end{tabular}

Nota: Elaboración propia.

\section{Nivel de desarrollo del pensamiento crítico dialógico del estudiantado}

En la dimensión dialógica del pensamiento crítico, al igual que en las anteriores, el estudiantado presenta un mayor porcentaje del nivel bajo 57,6\%, respecto al 6,8\% del nivel alto. Ver detalles en la Tabla 3.

Tabla 3: Nivel de pensamiento crítico dialógico del estudiantado

\begin{tabular}{ccc}
\hline Dialógico & $\mathrm{n}$ & $\%$ \\
\hline Alto & 17 & 6,8 \\
Medio & 89 & 35,6 \\
Bajo & 144 & 57,6 \\
\hline Total & 250 & $100 \%$ \\
\hline
\end{tabular}

Nota: Elaboración propia.

\section{Discusión}

El presente estudio determinó el nivel de desarrollo del pensamiento crítico del estudiantado de las instituciones educativas de educación primaria de Chimbote, año 2017. Analizando los hallazgos encontramos que más de la mitad del estudiantado presenta un nivel bajo de pensamiento crítico. La causa de estos resultados podría ser la enseñanza tradicional, la cual representa un serio problema, dado que al estudiantado se le proporciona mayor conocimiento y menor ejercicios de razonamiento, análisis, reflexión y divergencias en la construcción de su propio aprendizaje. En ese sentido, ahí la presencia de hechos demuestra el poco interés que tiene el estudiantado por leer, analizar críticamente, reflexionar sobre un tema, descubrir, afirmar lo que se dice o asume. 
http://doi.org/10.15359/ree.25-1.15

http://www.una.ac.cr/educare

educare@una.ac.cr

Sumado a esto, los pocos recursos didácticos con que cuenta el personal docente como guía y mediador del aprendizaje podrían haber permitido que la construcción de las competencias que promuevan en el estudiantado sobre el pensamiento crítico llegue a los niveles de abstracción inferior a los esperados, dejando débil la posibilidad de que el estudiantado sea impulsor de su propio aprendizaje. Son varias las razones por las cuales se tendría que analizar por qué no se logran alcanzar los parámetros más altos en las evaluaciones y el desarrollo del pensamiento crítico. Sin embargo, se debe seguir insistiendo en el uso de estrategias cognitivas y metacognitivas donde se desarrolle esa capacidad de inferir, argumentar, debatir, analizar, reflexionar y la búsqueda de solución de problemas, ya que ello promueve el desarrollo del pensamiento crítico. Al respecto, en un estudio (Yubero Jiménez y Larrañaga Rubio, 2010) se utilizaron cuestionarios de lectura y psicológicos con 1669 estudiantes del nivel primaria, los resultados que obtuvieron fue que la importancia de la lectura varía en función al comportamiento del estudiantado, de sus hábitos de lectura, de sus padres y madres, como también del contexto escolar y de aua maestros y maestras.

Respecto a la dimensión sustantiva del pensamiento crítico, el $65.6 \%$ de las personas participantes se encuentran en un nivel bajo. Un estudio hecho en el Callao (Milla Virhuez, 2012) reporta resultados similares a los nuestros, a pesar que su población de estudio fueron estudiantes de secundaria. De igual modo, este panorama es preocupante, posiblemente debido a que las tareas constructivas del personal docente son escasas y aún existe una enseñanza tradicional, y con la deficiencia de impartir nuevas estrategias para el desarrollo de habilidades de lectura en el estudiantado. Por ello, el educando requiere de elementos básicos en el ejercicio de la inteligencia para que sea capaz de confrontar realidades, polemizar, cuestionar y aportar a la solución con una visión distinta (Solé et al., 2005).

En cuanto a la dimensión dialógica del pensamiento crítico, más de la mitad del estudiantado presenta un nivel bajo de la dimensión dialógica. Esta dimensión se enfoca en el análisis o la integración de puntos de vista divergentes o en contraposición en relación con el suyo propio. Dados nuestros resultados, es necesario que se empleen estrategias en el ámbito pedagógico, donde el personal docente proponga situaciones significativas para el desarrollo del razonamiento en el estudiantado (Nosich, 2003). En ese sentido, existe un arduo trabajo por hacer en favor del estudiantado, donde muchas veces no pueden realizar comprensión referente a análisis, reflexión y criticidad de un tema. En ese sentido, es importante la participación del personal docente como facilitador en el aprendizaje del estudiantado.

El presente estudio utilizó solo un grado de educación primaria para el desarrollo del estudio, carece de validez externa, sin embargo, parte de esta limitación se trató utilizando un muestreo probabilístico. La fortaleza del presente se dio en utilizar un cuestionario validado en Perú, que se adaptó y contextualizó a un lenguaje propio. Para ello se aplicó una prueba piloto a una población distinta de la muestra, y se utilizaron técnicas estadísticas para evaluar la confiabilidad.

8 
http://doi.org/10.15359/ree.25-1.15

\section{Conclusión}

A partir de los resultados, podemos concluir que existe un déficit de pensamiento crítico en el estudiantado de educación primaria en las dimensiones sustantiva y dialógica. La existencia de esta debilidad conlleva a poner de relieve intervenciones educativas basadas en el uso de estrategias de aprendizaje activas, aplicables desde la práctica pedagógica en las instituciones educativas.

Por otro lado, se percibe que nuestra educación sigue siendo la misma a pesar del tiempo, los resultados demuestran la necesidad de implementar programas educativos aplicables desde el Currículo Nacional de la Educación Básica Regular, donde el personal docente sea capacitado para impulsar proyectos innovadores, desarrollo de la creatividad y la resolución de problemas, desarrollo de habilidades para la vida y así lograr enfrentar los desafíos que enfrenta en la sociedad.

La toma de decisiones para descomponer las rutinas del memorismo va a depender de las autoridades del futuro. Es urgente emprender una educación de base centrada en el estudiantado como respuesta a los desafíos en la movilización de los diferentes saberes y los retos a enfrentar en la sociedad.

Finalmente, es posible generar escenarios exploratorios e interactivos, pilotos, basados en la lectura, donde se fomente la tertulia literaria, se resalte información relevante, se argumente, se extraigan conclusiones, se debatan las ideas, se valore la igualdad en los diferentes hechos y actividades de lectura de apreciación, comentario e interpretación. Solo propuestas así de lectura permitirán establecer relaciones con el nuevo conocimiento.

\section{Agradecimiento}

El presente estudio ha sido financiado por la Universidad Católica Los Ángeles de Chimbote.

\section{Declaración de Material complementario}

Este artículo tiene disponible, como material complementario:

-La versión preprint del artículo en https://doi.org/10.5281/zenodo.3661217 
http://doi.org/10.15359/ree.25-1.15

http://www.una.ac.cr/educare

educare@una.ac.cr

\section{Referencias}

Bezanilla Albisua, M. J., Poblete Ruiz, M., Fernández Nogueira, D., Arranz Turnes, S. y Campo Carrasco, L. (2018). El pensamiento crítico desde la perspectiva de los docentes universitarios. Estudios Pedagógicos 44(1), 89-113. https://scielo.conicyt.cl/pdf/estped/ v44n1/0718-0705-estped-44-01-00089.pdf

Díaz-Larenas, C. H., Ossa-Cornejo, C. J., Palma-Luengo, M. R., Lagos-San Martín, N. G. y Boudon Araneda, J. I. (2019). El concepto de pensamiento crítico según estudiantes chilenos de pedagogía. Sophia, 27, 267-288. http://scielo.senescyt.gob.ec/scielo.php?script=sci arttext\&pid=S1390-86262019000200275\#ref1

Hernández Sampieri, R., Fernández Collado, C. y Baptista Lucio, M. (2014). Metodología de la Investigación (6. ${ }^{\mathrm{a}}$ ed.). Interamericana editores.

López Aymes, G. (2012). Pensamiento critico en el aula. Docencia e Investigación, 37(22), 41-60. http://educacion.to.uclm.es/pdf/revistaDI/3 22 2012.pdf

Marciales Vivas, G. P. (2003). Pensamiento crítico: Diferencias en estudiantes universitarios en el tipo de crrencias, estrategias e inferencias en la lectura crítica de textos [Tesis doctoral]. Universidad Complutense de Madrid. https://core.ac.uk/download/pdf/19709382.pdf

Milla Virhuez, M. R. (2012). Pensamiento crítico en estudiantes de quinto de secundaria de los colegios de Carmen de la Legua Callao [Tesis de maestría]. Universidad San Ignacio de Loyola, Lima, Perú. http://repositorio.usil.edu.pe/bitstream/123456789/1217/1/2012 Milla Pensamiento cr\%C3\%ADtico en estudiantes de quinto de secundaria.pdf

Ministerio de Educación. (2006). Guía para el desarrollo del pensamiento crítico. Autor. http:// es.slideshare.net/centropoblado3/gua-para-el-desarrollo-del-pensamiento-crticominedu

Ministerio de Educación. (2015a). Evaluación PISA 2015. http://umc.minedu.gob.pe/resultadosde-evaluacion-pisa-2015/

Ministerio de Educación. (2015b). Rutas de aprendizaje. http://www.minedu.gob.pe/rutas-delaprendizaje/primaria.php

Ministerio de Educación. (2016a). Currículo nacional de la educación básica. http://www.minedu. gob.pe/curriculo/pdf/curriculo-nacional-2016-2.pdf

Ministerio de Educación. (2016b). Resultados de la evaluación censal de estudiantes. ECE 2016. Autor. http://umc.minedu.gob.pe/evaluacion-censal-de-estudiantes-2016/ 
http://doi.org/10.15359/ree.25-1.15

http://www.una.ac.cr/educare educare@una.ac.cr

Nosich, G. M. (2003). Aprender a pensar. Pensamiento analítico para estudiantes. Pearson Educación.

Paul, R. y Elder, L. (2003) La mini-guía para el pensamiento crítico. Conceptos y herramientas. Fundación para el pensamiento crítico. https://www.criticalthinking.org/resources/PDF/ SP-ConceptsandTools.pdf

Prado Coronado, M. V. (2019). El pensamiento critico y su relación con el aprendizaje autónomo de los alumnos del 5to. de secundaria de la Red 03 de La Perla - Callao [Tesis Doctoral]. Universidad Nacional de Educación Enrique Guzmán y Valle, Lima, Perú. http://repositorio. une.edu.pe/handle/UNE/3888

Solé, I., Mateos, M., Miras, M., Martín, E., Castells, N., Cuevas, I. y Gràcia, M. (2005). Lectura, escritura y adquisición de conocimientos en educación secundaria y Educación Universitaria. Journal for the Study of Education and Development. Infancia y Aprendizaje, 28(3), 329-347. https://doi.org/10.1174/0210370054740241

Tamayo, O. E., Zona, R. y Zoaiza, Y. E. (2015). El pensamiento crítico en la eduacción. Algunas categorías centrales en su estudio. Revista Latinoamericna de Estudios Educativos, 11(2), 111-133. https://www.redalyc.org/pdf/1341/134146842006.pdf

Villa, A. y Poblete, M. (Dirs.). (2007). Aprendizaje basado en competencias. Una propuesta para la evaluación de las competencias genéricas. https://investigacion.udgvirtual.udg.mx/ personal/jdelgado/personal_page/lirbos_Chan/Aprendizaje\%20Basado\%20en\%20 Competencias.pdf

Yubero Jiménez, S. y Larrañaga Rubio, E. (2010). El valor de la lectura en relación con el comportamiento lector. Un estudio sobre los hábitos lectores y el estilo de vida en niños. Ocnos:RevistadeEstudios Sobre Lectura,6,7-20.https://doi.org/10.18239/ocnos 2010.06.01 